EPJ Web of Conferences 66, 09014 (2014)

DOI: $10.1051 /$ epjconf/20146609014

(C) Owned by the authors, published by EDP Sciences, 2014

\title{
The first precision measurement of deeply bound pionic states in ${ }^{121} \mathrm{Sn}$
}

Takahiro Nishi ${ }^{1, a}$, Georg P. A. Berg ${ }^{2}$, Masanori Dozono ${ }^{3}$, Hiroyuki Fujioka ${ }^{4}$, Naoki Fukuda ${ }^{3}$, Tatsuya Furuno ${ }^{4}$, Hans Geissel ${ }^{5}$, Ryugo S. Hayano ${ }^{1}$, Naoto Inabe ${ }^{3}$, Kenta Itahashi ${ }^{3}$, Satoshi Itoh ${ }^{1}$, Daisuke Kameda ${ }^{3}$, Toshiyuki Kubo ${ }^{3}$, Hiroaki Matsubara ${ }^{6}$, Shin'ichiro Michimasa ${ }^{7}$, Kenjiro Miki ${ }^{8}$, Hiroyuki Miya ${ }^{7}$, Yohei Murakami ${ }^{1}$, Masaki Nakamura ${ }^{3}$, Noritsugu Nakatsuka ${ }^{3}$, Shumpei Noji ${ }^{1}$, Kota Okochi ${ }^{1}$, Shinsuke Ota ${ }^{7}$, Hiroshi Suzuki ${ }^{3}$, Ken Suzuki ${ }^{9}$, Motonobu Takaki ${ }^{7}$, Hiroyuki Takeda ${ }^{3}$, Yoshiki K. Tanaka ${ }^{1}$, Koichi Todoroki ${ }^{1}$, Kyo Tsukada ${ }^{10}$, Tomohiro Uesaka ${ }^{3}$, Yuni N. Watanabe ${ }^{1}$, Helmut Weick ${ }^{5}$, Hiroyuki Yamada ${ }^{1}$, and Koichi Yoshida ${ }^{3}$

${ }^{1}$ Department of Physics, University of Tokyo, Tokyo, 113-0033, Japan

2 JINA and Department of Physics, University of Notre Dame, Indiana, 46556, USA

${ }^{3}$ Nishina Center for Accelerator-Based Science, RIKEN, Saitama, 351-0198, Japan

${ }^{4}$ Department of Physics, Kyoto University, Kyoto, 606-8502, Japan

${ }^{5}$ GSI Helmholtzzentrum für Schwerionenforschung GmbH, D-64291 Darmstadt, Germany

${ }^{6}$ National Institute of Radiological Sciences (NIRS), Chiba 263-8555, Japan

${ }^{7}$ Center of Nuclear Study, University of Tokyo, Tokyo, 113-0033, Japan

${ }^{8}$ Research Center for Nuclear Physics, Osaka University, 567-0047 Osaka, Japan

${ }^{9}$ Stefan-Meyer-Institut für subatomare Physik, 1090, Vienna, Austria

${ }^{10}$ Department of Physics, Tohoku University, 980-8578, Sendai, Japan

\begin{abstract}
We performed a precision missing-mass spectroscopy experiment of the deeply bound pionic states in a ${ }^{121} \mathrm{Sn}$ atom using the $\left(d,{ }^{3} \mathrm{He}\right)$ reaction near the $\pi^{-}$emission threshold. The experiment serves as a pilot experiment for our new 'pionic atom factory project' at RIBF, which aims at precision spectroscopy of the energy spectrum of the pionic atom of isotopes and isotones. The result of the pilot experiment demonstrated the potentiality of BigRIPS and of the RIBF facilities for the project. The current status of the analysis is reported.
\end{abstract}

\section{Introduction}

The chiral condensate $\langle q q\rangle$ is an order parameter of the chiral symmetry spontaneous breaking. The magnitude of the $\langle q q\rangle$ is expected to decrease according to the partial restoration of the chiral symmetry in a high temperature/density condition $[1,2]$. The experimental evaluation of the change of $\langle q q\rangle$ is one of the most important subjects in modern hadron physics. Recent studies suggested that the magnitude of the $\langle q q\rangle$ at the normal nuclear density, that is, at high density and low temperature condition, can be deduced by the isovector scattering length $b_{1}$ [3-5] in the pion-nuclear $s$-wave optical

ae-mail: nishi@nucl.phys.s.u-tokyo.ac.jp

This is an Open Access article distributed under the terms of the Creative Commons Attribution License 2.0, which permits unrestricted use, distribution, and reproduction in any medium, provided the original work is properly cited. 
potential of the Ericson-Ericson type. The potential is expressed as

$$
V_{s}(r)=-\frac{2 \pi}{\mu}\left[\epsilon_{1}\left\{b_{0} \rho(r)+b_{1} \delta \rho(r)\right\}+\epsilon_{2} B_{0} \rho(r)^{2}\right] .
$$

Here, $\rho$ and $\delta \rho$ mean $\rho_{n}+\rho_{p}$ and $\rho_{n}-\rho_{p}$, respectively, $\mu$ is the reduced mass, and $\epsilon_{1}, \epsilon_{2}$ represent the $1+m_{\pi} / M_{\text {nucleon }}$ and $m_{\pi} / 2 M_{\text {nucleon }}$, respectively.

The $b_{1}$ in the medium can be determined from the spectroscopy of a 'deeply-bound pionic atom' that is heavy atoms capturing a pion in deep (such as $1 s$ ) atomic orbits similar to the capture of an electron in a shell. So far at GSI the $1 s$ pionic states in ${ }^{205} \mathrm{~Pb}$ and ${ }^{115,119,123} \mathrm{Sn}$ have been discovered [610]. The deduced value of $b_{1}$ was compared with that of the vacuum, which was measured from the pionic hydrogen [11]. Although these data suggested partial chiral restoration, the evaluated value still had large systematic and statistical errors. The main part of the systematic error comes from the ambiguity of neutron density function in nuclei, because $b_{1}$ appears in the potential with $\delta \rho(r)$ as shown in eq (1).

In order to reduce these errors, we are planning the pionic Atom Factory (piAF) project, in which the deeply-bound pionic atoms of isotopes and isotones will be studied. We will perform the project at the RI Beam Factory (RIBF), RIKEN [12], where a high intensity ( $\approx 100 \mathrm{pnA})$ and high energy $\left(T_{d}\right.$ $=250 \mathrm{MeV} / \mathrm{u}$ ) deuteron beam, and the high resolving-power spectrometer BigRIPS are available.

\section{Pilot experiment}

A pilot experiment was carried out in October $2010[13,14]$ with ${ }^{122} \mathrm{Sn}$ target. The purpose of the experiment was to examine the whole experimental setup, including a newly-developed ion optics. We produced pionic states in ${ }^{121} \mathrm{Sn}$ by the ${ }^{121} \mathrm{Sn}\left(d,{ }^{3} \mathrm{He}\right)$ reaction near the $\pi^{-}$emission threshold and collected data for 3 days. The $\mathrm{Q}$ value was deduced by measuring the ${ }^{3} \mathrm{He}$ momentum and calculating the missing mass . In this experiment the recoil-free condition was satisfied by choosing the deuteron beam energy of $250 \mathrm{MeV} / \mathrm{u}$. Under this condition the zero angular momentum transfer is preferred, so that $\Delta \mathrm{L}=0$ reactions such as $(1 s)_{\pi^{-}} \otimes\left(3 s_{1 / 2}\right)_{n^{-1}}$, or sensitive states to s-wave potential, become dominant.

The beam impinged on the ${ }^{121} \mathrm{Sn}$ target at the $\mathrm{F} 0$ focal plane and ${ }^{3} \mathrm{He}$ was produced in the $\left(d,{ }^{3} \mathrm{He}\right)$ reaction. The momentum of ${ }^{3} \mathrm{He}$ was magnetically analyzed by a fragment separator, BigRIPS, and measured by two multi-wire drift chambers (MWDCs) at the F5 dispersive focal plane. The ${ }^{3} \mathrm{He}$ particles were identified by the time of flight and the energy loss using a plastic scintillator immediately after the MWDCs and another plastic scintillator at a focal plane at down stream. The count rate at the dispersive focal plane was about $100 \mathrm{~Hz}$ for ${ }^{3} \mathrm{He}$ particles and about $200 \mathrm{kHz}$ for break-up protons. In spite of this condition, we succeed to identify ${ }^{3} \mathrm{He}$ by scintillators clearly and to reconstruct the trajectories of ${ }^{3} \mathrm{He}$ at the dispersive focal plane by the two MWDCs as the left panel of Fig. 1.

We developed a new ion optical mode to apply the dispersion-matching method [15]. At RIBF, the momentum spread of the beam is about $\pm 0.1 \%$ and it affects the resolution of the spectroscopy. To cancel the effect of the primary beam momentum spread, the dispersion-matching method had to be applied. The details about the optics and the dispersion-matching method are described in Ref. [16].

The right panel of Fig. 1 shows a preliminary result for the acceptance-corrected Q-value spectrum obtained in this experiment. The red vertical dashed line in the figure represents the quasi-free $\pi^{-}$ production threshold. The $\mathrm{Q}$ value was calculated by the ${ }^{3} \mathrm{He}$ trajectory (horizontal position and horizontal/vertical angle) at the dispersive focal plane and the primary beam momentum, which was estimated from the NMR in the dipole magnets in the beam line. The relation between the position at the dispersive focal plane and the ${ }^{3} \mathrm{He}$ momentum was calibrated using the $p\left(d,{ }^{3} \mathrm{He}\right) \pi^{0}$ reaction peak 

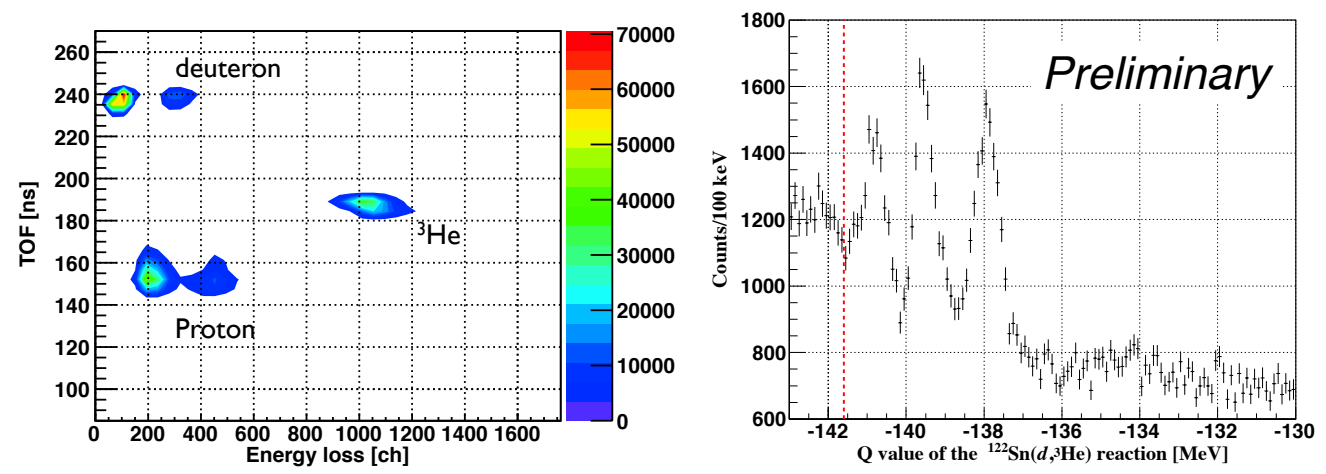

Figure 1. (Left) The time of flight and the energy loss measured by scintillators. The ${ }^{3} \mathrm{He}$ particles are clearly identified with excellent background rejection. (Right) The preliminary Q-value spectrum of the ${ }^{122} \mathrm{Sn}\left(d,{ }^{3} \mathrm{He}\right)$ reaction. The red vertical dashed line represents the quasi-free $\pi^{-}$production threshold.

with a polyethylene $\left(\mathrm{CH}_{2}\right)_{n}$ target. The higher order aberration in the ion optics and a position/angular acceptance were already corrected in the spectrum [16], although these corrections and momentum calibration are yet to be optimized.

\section{Discussion}

In the Q-value spectrum shown in Fig. 1, the peaks on the right side of the quasi-free $\pi^{-}$threshold represent the pionic bound states. Although the spectrum is yet to be finalized, three peaks are clearly observed. Each peak had a different angular dependence, which indicate different momentum transfers for the peaks. This is the first observation of the the pionic states in ${ }^{121} \mathrm{Sn}$ and of the angular distribution of the pionic state production cross section in the $\left(d,{ }^{3} \mathrm{He}\right)$ reaction. The measured spectrum is qualitatively in good agreement with theoretically-calculated spectrum [17], including the angular dependence of the production cross section.

\section{Conclusion and future perspectives}

In this pilot experiment, the pionic bound states and their angular dependence were observed for the first time in the short data-acquisition period of 3 days. This high-speed data acquisition is essential for systematic spectroscopy. The preliminary results are in good qualitative agreement with the theoretically calculated spectrum.

These results demonstrated a potentiality of BigRIPS and the RIBF facility for the 'pionic atom factory project', in which the deeply-bound pionic atoms of a wide range of isotopes and isotones will be produced. We are now finalizing the result of the pilot experiment to deduce the binding energies and widths of the pionic bound states. 


\section{Acknowledgements}

We would like to show our greatest appreciation for the support of the RIKEN Nishina Center for Accelerator-Based Science. This work is also supported by the Grants-in-Aid for Scientific Research (22105517, 20540273, 22105510), JSPS fellows (No. 232274), and Specially Promoted Research (20002003). G. P. A. Berg was supported by the NSF Grand PHYS0822648 for the Joint Institute for Nuclear Astrophysics (JINA).

\section{References}

[1] U. Vogl and W. Weise, Prog. Part. Nucl. Phys. 27, 195 (1991) and references therein.

[2] T. Hatsuda and T. Kunihiro, Phys. Rept. 247, 221 (1994) and references therein.

[3] E. E. Kolomeitsev, N. Kaiser, and W. Weise, Phys. Rev. Lett. 90, 092501 (2003).

[4] D. Jido, T. Hatsuda and T. Kunihiro, Phys. Lett. B670, 109 (2008).

[5] N. Ikeno et al., Prog. Theor. Phys., 126, 483 (2011).

[6] H. Gilg et al., Phys. Rev. C62, 025201 (2000).

[7] K. Itahashi et al., Phys. Rev. C62, 025202 (2000).

[8] H. Geissel et al., Phys. Rev. Lett. 88, 122301 (2002).

[9] H. Geissel et al., Phys. Lett. B 549, 64 (2002).

[10] K. Suzuki et al., Phys. Rev. Lett. 92, 072302 (2004).

[11] H.-Ch. Schröder et al., Eur. Phys. J. C21, 473 (2001).

[12] T. Kubo et al., Nucl. Instrum. Methods. in Phys. Res. B204, 97 (2003).

[13] S. Itoh et al., RIKEN Accel. Prog. Rep. 44, 221 (2011).

[14] T. Nishi et al., RIKEN Accel. Prog. Rep. 44, 24 (2011).

[15] T. Wakasa et al., Nucl. Instrum. Methods. in Phys. Res. A482, 79 (2002).

[16] T. Nishi et al., Nucl. Instrum. Methods. in Phys. Res. B, dx.doi.org/10.1016/j.nimb.2013.05.055 (2013).

[17] N. Ikeno, H. Nagahiro and S. Hirenzaki, Eur. Phys. J. A47, 161 (2011). 